\title{
Stellar yields - theory and observations
}

\section{Amanda I. Karakas}

Research School of Astronomy \& Astrophysics, the Australian National University, Canberra ACT, Australia

email: amanda.karakas@anu.edu.au

\begin{abstract}
Stellar yields are an essential tool for studies of chemical evolution. For low and intermediate-mass stars $\left(0.8\right.$ up to $\left.8-10 M_{\odot}\right)$ the richest nucleosynthesis occurs when the stars are on the asymptotic giant branch (AGB) of stellar evolution. We discuss the main nucleosynthesis outcomes, along with the uncertainties that affect the theoretical calculations. The uncertainties in the physics can be improved by comparing theoretical models to observations, including chemically peculiar metal-poor stars, along with AGB stars and their progeny.
\end{abstract}

Keywords. stars: abundances, evolution, AGB and post-AGB, carbon, Galaxy: abundances, galaxies: abundances

\section{Introduction}

Stars with initial masses between $0.8-8 M_{\odot}$ evolve through core hydrogen and helium burning before ascending the asymptotic giant branch (AGB). The exact mass range of AGB stars is metallicity dependent, with the minimum mass for core helium/carbon burning decreasing with decreasing metallicity. The AGB phase is the last nuclear burning phase for these low and intermediate-mass stars.

AGB stars are important for the lives of galaxies because they can produce substantial amounts of the gas and dust (Sloan et al. 2008). Galaxies dominated by intermediateage stellar populations have a significant fraction of their starlight emitted by low and intermediate mass stars, especially when they evolve off the main sequence to the giant branches (e.g., Maraston 2005). AGB stars in particular are bright and therefore observable in resolved stellar populations in nearby galaxies (e.g., Whitelock et al. 2013).

AGB stars can produce a rich array of nucleosynthesis products including carbon and roughly half of all elements heavier than iron by the slow neutron capture process (the $s$ process, e.g., Busso et al. 1999; Herwig 2005; Romano et al. 2010; Kobayashi et al. 2011). The other half of heavy elements are produced by the rapid neutron capture process, which likely occurs in core-collapse supernova (e.g., Arnould et al. 2007). Intermediatemass stars over about $4 M_{\odot}$ experience hot hydrogen burning (HBB) at the base of their convective envelopes, which produces $\mathrm{Li}, \mathrm{N}, \mathrm{Na}$ and $\mathrm{Al}$. It is for this last reason that intermediate-mass AGB stars have been considered polluters of Galactic globular clusters (e.g., Ventura \& D'Antona 2009).

\section{Stellar yields}

The stellar evolution of low and intermediate-mass stars prior to and during the thermally-pulsing AGB phase has been discussed elsewhere (e.g., Karakas \& Lattanzio 2014). The main point is that hydrogen and helium nucleosynthesis occurs in the core, some of which may be mixed into the envelope. The main nucleosynthesis contributions of stars with masses up to $\approx 4 M_{\odot}$ includes $\mathrm{C}, \mathrm{N}, \mathrm{F}$, and elements produced by the $s$-process including $\mathrm{Sr}, \mathrm{Y}, \mathrm{Ba}, \mathrm{La}$, and $\mathrm{Pb}$. For stars above this mass limit, $\mathrm{HBB}$ occurs 
and shapes the stellar yields. The main nucleosynthesis for these intermediate-mass stars includes enrichments in $\mathrm{He}, \mathrm{Li},{ }^{13} \mathrm{C}, \mathrm{N}$, and possibly $\mathrm{Na}, \mathrm{Mg}$, and $\mathrm{Al}$ depending on the input physics and numerical details of the stellar evolutionary code used to calculate the models (e.g., Ventura \& D'Antona 2005; Karakas 2010).

Stellar yields are an essential ingredient of chemical evolution models. Stellar yields of AGB stars focus either on large grids of stellar models covering a significant portion of the mass and metallicity range appropriate for chemical evolution modelling (see Karakas \& Lattanzio 2014 for list of yields available). There are however crucial gaps in our knowledge. These gaps are most apparent for AGB stars of low metallicity (e.g., $[\mathrm{Fe} / \mathrm{H}]$ $\leqslant-3)$ and for the production of $s$-process elements for all mass and metallicity ranges.

The evolution and nucleosynthesis of low and intermediate-mass stars is seriously affected by numerical modelling uncertainties as well as uncertainties in the input physics. We refer to previous reviews on AGB stars for a thorough discussion of this topic (e.g., Herwig 2005; Karakas \& Lattanzio 2014). The main uncertainties include convection and the treatment of convective borders, which influences the efficiency of the mixing, as well as the formation of ${ }^{13} \mathrm{C}$ pockets in the He-intershell, a necessary ingredient in the synthesis of $s$-process elements in low-mass AGB stars (e.g., Busso et al. 1999). Mass loss is also another key uncertainty, as it determines the AGB lifetime.

Observations of AGB stars and their progeny can be used to constrain theoretical models. For example, carbon enhanced metal-poor stars with $s$-process enrichments can be used to constrain models of metal-poor AGB stars (e.g., Lugaro et al. 2012). Observations of the brightest AGB stars can provide key information on the efficiency of mixing and $s$-process element production in stars at the maximum mass to experience the AGB phase (e.g., van Raai et al. 2012).

Significant progress has been made over the past decade to improve our understanding of the AGB phase of stellar evolution. Even so, there are significant gaps in available tables of stellar yields as well as uncertainties that affect the stellar yield calculations. The uncertainties in turn affect the accuracy and reliability of chemical evolution model predictions (e.g., Romano et al. 2010). Theoretical effort is needed to address these issues, especially because current and future surveys (e.g., SEGUE, GALAH, APOGEE, and GAIA-ESO) will provide stellar abundance data for hundreds of thousands of stars in our Milky Way Galaxy. Stellar yields from all mass ranges will be needed for the interpretation.

\section{References}

Arnould, M., Goriely, S., \& Takahashi, K. 2008, Physics Reports, 450, 97

Busso, M., Gallino, R., \& Wasserburg, G. J. 1999, ARAA, 37, 239

Herwig, F. 2005, ARAA, 43, 435

Karakas, A. I. 2010, MNRAS, 403, 1413

Karakas, A. I. \& Lattanzio, J. C. 2014, PASA, 31, e30

Kobayashi, C., Karakas, A. I., \& Umeda, H. 2011, MNRAS, 414, 3231

Lugaro, M., Karakas, A. I., Stancliffe, R. J., \& Rijs, C. 2012, ApJ, 747, 2

Maraston, C. 2005, MNRAS, 362, 799

Romano, D., Karakas, A. I., Tosi, M., \& Matteucci, F. 2010, A\&A, 522, A32

Sloan, G. et al. 2008, ApJ, 686, 1056

van Raai, et al. 2012, A\&A, 540, A44

Ventura, P. \& D'Antona, F. 2005, A\& $A, 431,279$

Ventura, P. \& D'Antona, F. 2009, A\&A A, 499, 835

Whitelock, P. A. et al. 2013, MNRAS, 428, 2216 